\title{
Some Remarks on Multiplicity Codes
}

\author{
Swastik Kopparty*
}

October 20, 2013

To Ilya Dumer, on the occasion of his 60th birthday

\begin{abstract}
Multiplicity codes are algebraic error-correcting codes generalizing classical polynomial evaluation codes, and are based on evaluating polynomials and their derivatives. This small augmentation confers upon them better local decoding, list-decoding and local list-decoding algorithms than their classical counterparts. We survey what is known about these codes, present some variations and improvements, and finally list some interesting open problems.
\end{abstract}

\footnotetext{
*Department of Mathematics \& Department of Computer Science, Rutgers University. swastik.kopparty@rutgers.edu. Research supported in part by a Sloan Fellowship and NSF CCF-1253886.
} 


\section{Introduction}

Reed-Solomon codes and Reed-Muller codes are classical families of error-correcting codes which have been widely influential in coding theory, combinatorics and theoretical computer science. These codes are based on evaluations of polynomials: a codeword of one of these codes is obtained by evaluating a polynomial over a finite field $\mathbb{F}_{q}$ of degree at most $d$ at all points in $\mathbb{F}_{q}^{m}$.

Multiplicity codes are a family of recently-introduced algebraic error-correcting codes based on evaluations of polynomials and their derivatives. Specifically, a codeword of a multiplicity code is obtained by evaluating a polynomial of degree at most $d$, along with all its derivatives of order $<s$, at all points in $\mathbb{F}_{q}^{m}$.

The $s=1$ versions of multiplicity codes are thus the classical Reed-Solomon $(m=1)$ and Reed-Muller $(m \geq 1)$ codes. We will see that by allowing $s$ to be larger than 1 , in many senses general multiplicity codes go beyond their $s=1$ counterparts.

Multiplicity codes with $m=1$ (i.e., based on univariate polynomials) were first considered by Rosenbloom and Tsfasman [RT97, who studied them for the purposes of producing optimal codes for the "M metric" (now known as the Rosenbloom-Tsfasman metric). They were also studied by Nielsen [Nie01, who showed that they admit list-decoding algorithms upto the Johnson bound, similar to the Reed-Solomon codes.

Multiplicity codes with general $m, s$ were defined by Kopparty, Saraf and Yekhanin KSY11. The main result of [KSY11] was that for every $\epsilon, \alpha>0$, for all $k$, there are multiplicity codes of dimension $k$, rate $1-\alpha$, and which are locally decodable from a constant fraction of errors with in just $O_{\epsilon, \alpha}\left(k^{\epsilon}\right)$ time. Prior to KSY11, codes with nontrivial local decoding algorithms were known only at rate $R<1 / 2$, and achieving local decoding complexity $O\left(k^{\epsilon}\right)$ required the code to have rather small rate $R=\epsilon^{(1 / \epsilon)}$ (the codes that were known to achieve these parameters were the Reed-Muller codes). It should be noted that more recent results have shown how to construct codes achieving parameters similar to those of multiplicity codes using significantly different ideas: Guo-Kopparty-Sudan [GKS13], Guo [Guo13] and Hemenway-Ostrovsky-Wooters [HOW13].

Subsequently, Guruswami-Wang GW11 and Kopparty Kop12 studied the list-decoding of univariate multiplicity codes, and showed that there are sequences of univariate multiplicity codes of rate $R$, list-decodable from $1-R-\epsilon$ fraction errors in polynomial time (achieving the so-called list-decoding capacity, thus providing another route to such codes after the breakthrough results of Parvaresh-Vardy [PV05] and GuruswamiRudra GR08]).

Global decoding of multivariate multiplicity codes was also considered in Kop12. There it was shown that multivariate multiplicity codes can be decoded upto half their minimum distance in polynomial time, and can be list-decoded from the Johnson bound in polynomial time.

The primary purpose of this paper is to survey the state of the art algorithms for dealing with multiplicity codes. Along the way we note some variations and improvements. Specifically:

1. We give an improved local decoding algorithm for multiplicity codes. The original local decoding algorithm of KSY11 for multiplicity codes worked as follows: in order to recover the correct value of the multiplicity codeword at a point $\mathbf{a} \in \mathbb{F}_{q}^{m}$, one would take $s^{O(m)}$ random lines in $\mathbb{F}_{q}^{m}$ passing through a, query the codeword on all those lines, and use the answer to decode the correct value at $\mathbf{a}$. Our improved local decoding algorithm is based on queries only $\exp (m)$ random lines through $\mathbf{a}$.

This new algorithm is based on two new ideas. First, we show that one can extract much more information from each line about the correct value at $\mathbf{a}$ than what the previous algorithm took advantage of. Second, we use a more sophisticated way of combining information from the different lines. For the previous algorithm, the problem of combining information from the various lines through a to recover the correct value of the codeword at a amounted to the problem of decoding a Reed-Muller code. In the new algorithm, this problem turns out to be a case of decoding a multiplicity code! 
2. The above framework admits a number of variations that could potentially be interesting for their own sake.

One variation leads to a "polynomial rate" constant-query error-correction scheme as follows: a message $\sigma \in \Sigma_{0}^{n}$, where $\left|\Sigma_{0}\right|=\exp (n)$, gets encoded into a codeword $c \in \Sigma^{n}$, where $\log |\Sigma|=n^{\epsilon} \cdot \log \left|\Sigma_{0}\right|$, such that even if a constant fraction of the coordinates of $c$ are corrupted, for any given 1$] \in[n]$ one can recover $\sigma_{i}$ with high probability using only $O(1)$ queries into $c$. Such large alphabet error-correction schemes were considered by Beimel and Ishai BI01.

Another variation allows local correction for some low rate multiplicity codes using only $m$ lines, with a much simpler local correction algorithm.

3. Using ideas from the above improvements, we give a new algorithm for (global) decoding of multivariate multiplicity codes. The original approach of Kop12 was based on a family of $s^{O(m)}$ space filling curves that passed through all the points of $\mathbb{F}_{q}^{m}$. The new algorithm uses only $\exp (m)$ many curves. The property of the $s^{O(m)}$ curves used in Kop12 was "algebraic repulsion": no nonzero polynomial $P\left(X_{1}, \ldots, X_{m}\right)$ of moderate degree can vanish on all these curves. The family of curves that we use in this paper can be smaller because we require a weaker property: no nonzero polynomial $P\left(X_{1}, \ldots, X_{m}\right)$ of moderate degree can vanish on all these curves with high multiplicity.

4. We observe that encoding and unique decoding algorithms for multiplicity codes can be implemented in near-linear time (i.e., they run in time $O\left(n \cdot(\log n)^{O(1)}\right)$ ). For $m=1$, this follows from algorithms nearly identical to the ones from the classical univariate $(s=m=1)$ case, and for general $m$ it follows by refining a reduction to the $m=1$ case given in Kop12.

5. We gather a number of open questions and possible future research directions for the study of multiplicity codes.

Organization of this paper: In the next section we formally define multiplicity codes and state their basic properties. In Section 3 we discuss decoding algorithms for univariate multiplicity codes. In Section 4 we discuss decoding algorithms for multivariate multiplicity codes. In Section 5 we discuss encoding algorithms. We conclude with some discussion and open questions.

\section{Multiplicity Codes}

We begin with some general preliminaries on codes, polynomials and derivatives, and then move on to state the basic definitions and results about multiplicity codes.

\subsection{Codes}

Let $\Sigma$ be a finite set and let $n$ be an integer. We will work with $\Sigma^{n}$ equipped with the (normalized) Hamming metric $\Delta$, defined by:

$$
\Delta(x, y)=\operatorname{Pr}_{i \in[n]}\left[x_{i} \neq y_{i}\right] .
$$

A code of length $n$ over the alphabet $\Sigma$ is a subset $\mathcal{C}$ of $\Sigma^{n}$. The rate of the code is defined to be:

$$
R=\frac{\log _{|\Sigma|}|\mathcal{C}|}{n}
$$

The minimum distance of the code $\mathcal{C}$ is defined to be the smallest value $\delta$ of $\Delta\left(c, c^{\prime}\right)$ for distinct elements $c, c^{\prime}$ of $\mathcal{C}$.

\footnotetext{
${ }^{1}$ We use $[n]$ to denote the set $\{1,2, \ldots, n\}$.
} 
Encoding If $\mathcal{C} \subseteq \Sigma^{n}$ is a code, an encoding map for $\mathcal{C}$ is a bijection $E: \Sigma_{0}^{k} \rightarrow \mathcal{C}$ for some integer $k$. Often $\Sigma_{0}=\Sigma$, but it need not be. It will be important that this map $E$ is efficiently computable and efficiently invertible.

Unique Decoding In the problem of unique decoding the code $\mathcal{C}$ from $\eta$-fraction errors, where $\eta \leq \delta / 2$, we are given as input $r \in \Sigma^{n}$, and we wish to compute the unique $c \in \mathcal{C}$ (if any) such that $\Delta(r, c)<\eta$. The uniqueness follows from our condition relating $\eta$ and $\delta$.

List-Decoding In the problem of list-decoding the code $\mathcal{C}$ from $\eta$-fraction errors, we are given as input $r \in \Sigma^{n}$, and we wish to compute the set

$$
\mathcal{L}=\{c \in \mathcal{C} \mid \Delta(r, c)<\eta\}
$$

The maximum possible value of $|\mathcal{L}|$ as $r$ varies over all elements of $\Sigma^{n}$ is called the list-size for list-decoding $\mathcal{C}$ from $\eta$ fraction errors.

Local Correction and Local Decoding In the problem of locally correcting the code $\mathcal{C}$ from $\eta$-fraction errors, where $\eta \leq \delta / 2$, we are given oracle access to a string $r \in \Sigma^{n}$, and given as input $i \in[n]$, and we wish to compute $c_{i}$ for the unique $c \in \mathcal{C}$ (if any) such that $\Delta(r, c)<\eta$. The query complexity of such a local correction algorithm is the number of queries made to $r$; both the query complexity and time complexity could potentially be sublinear in $n$ (and indeed this is the interesting case).

For local decoding, we deal with a code $\mathcal{C}$ along with an encoding map $E: \Sigma_{0}^{k} \rightarrow \mathcal{C}$. In the problem of local decoding $(\mathcal{C}, E)$ from $\eta$-fraction errors, where $\eta \leq \delta / 2$, we are given oracle access to $r \in \Sigma^{n}$, and input $i \in[k]$, and we wish to compute $x_{i}$ for the unique $x \in \Sigma_{0}^{k}$ (if any) such that $\Delta(r, E(x))<\eta$. The query complexity of such a local decoding algorithm is the number of queries made to $r$; again, both the query complexity and time complexity could potentially be sublinear in $n$ (and indeed this is the interesting case).

The difference between local decoding and local correction is that in local decoding, we are trying to recover symbols of the original message, while in local correction, we are trying to recover symbols of the codeword.

\subsection{Polynomials and Derivatives}

For a vector $\mathbf{i}=\left\langle i_{1}, \ldots, i_{m}\right\rangle$ of non-negative integers, its weight, denoted wt(i), equals $\sum_{j=1}^{m} i_{j}$.

For a field $\mathbb{F}$, let $\mathbb{F}\left[X_{1}, \ldots, X_{m}\right]=\mathbb{F}[\mathbf{X}]$ be the ring of polynomials in the variables $X_{1}, \ldots, X_{m}$ with coefficients in $\mathbb{F}$. For a vector of non-negative integers $\mathbf{i}=\left\langle i_{1}, \ldots, i_{m}\right\rangle$, let $\mathbf{X}^{\mathbf{i}}$ denote the monomial $\prod_{j=1}^{m} X_{j}^{i_{j}} \in \mathbb{F}[\mathbf{X}]$.

We now define derivatives and the multiplicity of vanishing at a point.

Definition 1 ((Hasse) Derivative) For $P(\mathbf{X}) \in \mathbb{F}[\mathbf{X}]$ and non-negative vector $\mathbf{i}$, the $\mathbf{i}$ th (Hasse) derivative of $P$, denoted $P^{(\mathbf{i})}(\mathbf{X})$, is the coefficient of $\mathbf{Z}^{\mathbf{i}}$ in the polynomial $\tilde{P}(\mathbf{X}, \mathbf{Z}) \stackrel{\text { def }}{=} P(\mathbf{X}+\mathbf{Z}) \in \mathbb{F}[\mathbf{X}, \mathbf{Z}]$.

Thus,

$$
P(\mathbf{X}+\mathbf{Z})=\sum_{\mathbf{i}} P^{(\mathbf{i})}(\mathbf{X}) \mathbf{Z}^{\mathbf{i}}
$$

We will need some basic properties of the Hasse derivative (see [HKT08]).

Proposition 2 (Basic properties of Hasse derivatives) Let $P(\mathbf{X}), Q(\mathbf{X}) \in \mathbb{F}[\mathbf{X}]^{m}$ and let $\mathbf{i}$, $\mathbf{j}$ be vectors of nonnegative integers. Then: 
1. $P^{(\mathbf{i})}(\mathbf{X})+Q^{(\mathbf{i})}(\mathbf{X})=(P+Q)^{(\mathbf{i})}(\mathbf{X})$.

2. $(P \cdot Q)^{(\mathbf{i})}(\mathbf{X})=\sum_{0 \leq \mathbf{e} \leq \mathbf{i}} P^{(\mathbf{e})}(\mathbf{X}) \cdot Q^{(\mathbf{i}-\mathbf{e})}(\mathbf{X})$.

3. $\left(P^{(\mathbf{i})}\right)^{(\mathbf{j})}(\mathbf{X})=\left(\begin{array}{c}\mathbf{i}+\mathbf{j} \\ \mathbf{i}\end{array}\right) P^{(\mathbf{i}+\mathbf{j})}(\mathbf{X})$.

Definition 3 (Multiplicity) For $P(\mathbf{X}) \in \mathbb{F}[\mathbf{X}]$ and $\mathbf{a} \in \mathbb{F}^{m}$, the multiplicity of $P$ at $\mathbf{a} \in \mathbb{F}^{m}$, denoted mult $(P, \mathbf{a})$, is the largest integer $M$ such that for every non-negative vector $\mathbf{i}$ with $\operatorname{wt}(\mathbf{i})<M$, we have $P^{(\mathbf{i})}(\mathbf{a})=0$ (if $M$ may be taken arbitrarily large, we set $\operatorname{mult}(P, \mathbf{a})=\infty$ ).

Next, we state a basic bound on the total number of zeroes (counting multiplicity) that a polynomial can have on a product set $S^{m}$. An elementary proof of this lemma can be found in [DKSS09].

Lemma 4 Let $P \in \mathbb{F}[\mathbf{X}]$ be a nonzero polynomial of total degree at most $d$. Then for any finite $S \subseteq \mathbb{F}$,

$$
\sum_{\mathbf{a} \in S^{m}} \operatorname{mult}(P, \mathbf{a}) \leq d \cdot|S|^{m-1} .
$$

In particular, for any integer $s>0$,

$$
\operatorname{Pr}_{\mathbf{a} \in S^{m}}[\operatorname{mult}(P, \mathbf{a}) \geq s] \leq \frac{d}{s|S|}
$$

\subsection{Multiplicity Codes}

Finally, we come to the definition of multiplicity codes.

Definition 5 (Multiplicity code [KSY11]) Let $s, d, m$ be nonnegative integers and let $q$ be a prime power. Let $\left.\Sigma=\mathbb{F}_{q}^{(m+s-1}\right)=\mathbb{F}_{q}^{\{\mathbf{i}: \mathrm{wt}(\mathbf{i})<s\}}$. For $P\left(X_{1}, \ldots, X_{m}\right) \in \mathbb{F}_{q}\left[X_{1}, \ldots, X_{m}\right]$, we define the order $s$ evaluation of $P$ at $\mathbf{a}$, denoted $P^{(<s)}(\mathbf{a})$, to be the vector $\left\langle P^{(\mathbf{i})}(\mathbf{a})\right\rangle_{\mathrm{wt}(\mathbf{i})<s} \in \Sigma$.

The multiplicity code of order-s evaluations of degree-d polynomials in $m$ variables over $\mathbb{F}_{q}$ is defined as follows. The code is over the alphabet $\Sigma$, and has length $q^{m}$ (where the coordinates are indexed by elements of $\left.\mathbb{F}_{q}^{m}\right)$. For each polynomial $P(\mathbf{X}) \in \mathbb{F}_{q}\left[X_{1}, \ldots, X_{m}\right]$ with $\operatorname{deg}(P) \leq d$, there is a codeword in $\mathcal{C}$ given by:

$$
\operatorname{Enc}_{s, d, m, q}(P)=\left\langle P^{(<s)}(\mathbf{a})\right\rangle_{\mathbf{a} \in \mathbb{F}_{q}^{m}} \in(\Sigma)^{q^{m}} .
$$

Technically speaking, we have only defined the multiplicity code as a subset of $\Sigma^{\mathbb{F}_{q}^{m}}$, without specifying an encoding map. We postpone the choice of a good encoding map to a later section.

Lemma 6 (Rate and distance of multiplicity codes [KSY11]) Let $\mathcal{C}$ be the multiplicity code of order $s$ evaluations of degree d polynomials in $m$ variables over $\mathbb{F}_{q}$. Then $\mathcal{C}$ has minimum distance at least $\delta=1-\frac{d}{s q}$ and rate $\frac{\left(\begin{array}{c}d+m \\ m\end{array}\right)}{\left(\begin{array}{c}s+m-1 \\ m\end{array}\right) q^{m}}$, which is at least

$$
\left(\frac{s}{m+s}\right)^{m} \cdot\left(\frac{d}{s q}\right)^{m} \geq\left(1-\frac{m^{2}}{s}\right)(1-\delta)^{m}
$$

We usually think of $m$ and $s$ as large constants (significantly smaller than $q$ ), and in light of the above parameters, having $s \gg m^{2}$ is particularly interesting. For the rest of this paper, when we speak of nearlinear time algorithms, this assumes that $m$ and $s$ are constants, and that $q$ and the blocklength $q^{m}$ tend to $\infty$.

One can easily convert such codes into codes over a constant sized (and even binary) alphabet via concatenation, while preserving the local decoding/correction properties. For details, see KSY11. 


\section{Decoding Univariate Multiplicity Codes}

We begin by discussing decoding of univariate multiplicity codes.

\subsection{Unique Decoding}

The classic Berlekamp-Welch algorithm for decoding Reed-Solomon codes up to half the minimum distance has a simple generalization to the case of univariate multiplicity codes. This generalization was first discovered by Nielsen $\left[\mathrm{Nie}_{1}\right]^{2}$. In fact, Nielsen showed how to do list-decoding of univariate multiplicity codes, discussed next.

Let us set the problem up. Recall that the alphabet for this code is $\mathbb{F}_{q}^{s}$. Thus the received word is a function $r: \mathbb{F}_{q} \rightarrow \mathbb{F}_{q}^{s}$. Abusing notation, we view this as a tuple of $s$ functions $r^{(i)}: \mathbb{F}_{q} \rightarrow \mathbb{F}_{q}$ for $0 \leq i<s$. We wish to find the unique $P(X)$ such that $\Delta\left(\operatorname{Enc}_{s, d, 1, q}(P), r\right)<\delta / 2$.

The algorithm tries to find an error-locator polynomial $E(X)$ and another polynomial $N(X)$, such that $N(X)=E(X) \cdot P(X)$.

- Search for nonzero polynomials $E(X), N(X)$ of degrees at most $(s q-d) / 2,(s q+d) / 2$ respectively such that for each $x \in \mathbb{F}_{q}$, we have the following equations:

$$
\begin{aligned}
N(x) & =E(x) r^{(0)}(x) \\
N^{(1)}(x) & =E(x) r^{(1)}(x)+E^{(1)}(x) r^{(0)}(x) \\
& \cdots \\
N^{(s-1)}(x) & =\sum_{i=0}^{s-1} E^{(i)}(x) r^{(s-1-i)}(x)
\end{aligned}
$$

This is a collection of $s q$ homogeneous linear equations in $(s q-d) / 2+1+(s q+d) / 2+1>s q$ unknowns (the coefficients of $E$ and $N$ ). Thus a nonzero solution $E(X), N(X)$ exists. Take any such nonzero solution.

- Given $E(X), N(X)$ as above, output $\frac{N(X)}{E(X)}$.

The analysis proceeds by showing that $N(X)-P(X) E(X)$, which is a degree $(s q+d) / 2$ polynomial, has $>(s q+d) / 2 s$ zeroes of multiplicity $\geq s$, and is thus the zero polynomial. This implies that $P(X)=$ $N(X) / E(X)$, and so $P(X)$ is the output of the algorithm, as desired.

\subsubsection{Unique decoding in near-linear time}

In this subsection we describe how to implement the above algorithm in near-linear time. The presentation follows the description of a near-linear time implementation of the Berlekamp-Welch algorithm in Sudan's lecture notes Sud01.

Let $R(X)$ be the unique polynomial of degree at most $s q-1$ such that for each $\alpha \in \mathbb{F}_{q}$ and $i<s$,

$$
R^{(<s)}(\alpha)=r^{(<s)}(\alpha) .
$$

\footnotetext{
${ }^{2}$ Nielsen's theorem analyzes the decoding radius in terms of the $m$-metric, and implies the decoding algorithms for the Hamming metric considered here.
} 
Such an $R(X)$ can be found in near-linear time by the classical Hermite interpolation algorithm of Chin Chi76. If $E(X)$ and $N(X)$ satisfy the equations (2), then we have that $N(X)-E(X) R(X)$ vanishes at each $x \in \mathbb{F}_{q}$ with multiplicity at least $s$. Thus:

$$
N(X)=E(X) R(X)-C(X) \cdot\left(X^{q}-X\right)^{s},
$$

for some $C(X) \in \mathbb{F}_{q}[X]$. Equivalently,

$$
\frac{N(X)}{E(X)\left(X^{q}-X\right)^{s}}=\frac{R(X)}{\left(X^{q}-X\right)^{s}}-\frac{C(X)}{E(X)} .
$$

Thus we are looking for $C(X), E(X)$ such that:

1. $\operatorname{deg}(E(X)) \leq(s q-d) / 2$,

2. the rational function $\frac{C(X)}{E(X)}$ approximates the rational function $\frac{R(X)}{\left(X^{q}-X\right)^{s}}$, in the sense that the numerator of their difference $N(X)=R(X) E(X)-C(X)\left(X^{q}-X\right)^{s}$ has degree at most $(s q+d) / 2$.

This problem can be solved in near-linear time via Strassen's continued fraction algorithm [Str81]. In fact, one can minimize the degree of $N(X)$ subject to the constraint that $\operatorname{deg}(E(X)) \leq(s q-d) / 2$.

Finally, the division step can also be performed in near-linear time. This completes the description of the near-linear time implementation of the unique decoder for univariate multiplicity codes.

\subsection{List-Decoding}

We now discuss the list-decoding of univariate multiplicity codes. Here we consider the problem of decoding from a fraction of errors which may be larger than half the minimum distance $\delta$.

By the Johnson bound, we know that for list-decoding univariate multiplicity codes from $(1-\sqrt{1-\delta})$ fraction errors, the list-size is at most poly $(q)$ (this only uses the fact that the distance of the code is $\geq \delta$ ). It is thus reasonable to ask whether there is a polynomial time algorithm to list-decode univariate multiplicity codes from $(1-\sqrt{1-\delta})$-fraction error.

In [Nie01, Nielsen gave such an algorithm. His algorithm generalizes the Guruswami-Sudan algorithm for list-decoding Reed-Solomon codes, and is also based on interpolation and root-finding.

Given a received word $r: \mathbb{F}_{q} \rightarrow \mathbb{F}_{q}^{s}$, one first interpolates a low-degree bivariate polynomial $Q(X, Y) \in$ $\mathbb{F}_{q}[X, Y]$ such that for each $\alpha \in \mathbb{F}_{q}$, the polynomial $Q\left(X, \sum_{j=0}^{s-1} r^{(j)}(\alpha)(X-\alpha)^{j}\right)$ vanishes with high multiplicity at $X=\alpha$. One then shows that every $P(X) \in \mathbb{F}_{q}[X]$ of degree at most $d$ with $\Delta\left(\operatorname{Enc}_{s, d, 1, q}(P), r\right) \leq$ $1-\sqrt{1-\delta}$, we have $Q(X, P(X))=0$. Finally, one can find all polynomials $P(X)$ satisfying this latter equation.

Recently Guruswami-Wang GW11] and Kopparty [Kop12 independently found improved results for listdecoding univariate multiplicity codes over prime fields.

The main result of GW11] is that order $s$ univariate multiplicity codes of distance $\delta$ over prime fields can, for every integer $0 \leq t<s$, be list-decoded from $\eta_{t}$ fraction errors with list-size at most $q^{O(s)}$, where:

$$
\eta_{t}=\frac{t+1}{t+2}\left(\delta-\frac{t}{s-t}\right)
$$

For $t=0$, the algorithm boils down to Nielsen's version of the Berlekamp-Welch algorithm for uniquedecoding multiplicity codes. 
The main result of Kop12 is that order $s$ univariate multiplicity codes of distance $\delta$ over prime fields can, for every integer $0 \leq t<s$ be list-decoded from $\eta_{t}^{\prime}$ fraction errors with list-size at most $q^{O(t s)}$, where:

$$
\eta_{t}^{\prime}=1-\left(\left(1-\frac{t}{s-t}\right) \cdot(1-\delta)\right)^{\frac{t+1}{t+2}}
$$

For $t=0$, the algorithm boils down to Nielsen's version of the Guruswami-Sudan algorithm for list-decoding univariate multiplicity codes.

Both these algorithms are based on deriving an order $t$ differential equation of the form:

$$
Q\left(X, P(X), P^{(1)}(X), \ldots, P^{(t-1)}(X)\right)=0
$$

from the received word $r$, such that every $P$ whose encoding is close to $r$ must satisfy this differential equation. In the algorithm of GW11 this differential equation is a linear differential equation, and in the algorithm of Kop12 this equation is a polynomial differential equation. These differential equations are then solved using Hensel-lifting / power series. See GW11 and Kop12 for the details. The decoding radius $\eta_{r}^{\prime}$ is always greater than $\eta_{r}$, but the algorithm and analysis of KKop12 are also more involved than that of GW11.

It is well known that the maximimum fraction of errors $\eta$ from which a code of rate $R$ and block-length $n$ can be list-decoded from while still having $\operatorname{poly}(n)$ list-size is $1-R-\epsilon$ (for arbitrarily small $\epsilon>0$ ). A code which achieves this is said to achieve list-decoding capacity. The first constructions of codes which achieved list-decoding capacity came from the breakthrough results of Parvaresh-Vardy PV05] and GuruswamiRudra GR08]. The above-mentioned results of [GW11] and Kop12 show that univariate multiplicity codes over prime fields achieve list-decoding capacity for every $R \in(0,1)$. This follows by noting that for univariate multiplicity codes, $R=1-\delta$, and that for every $\delta$, if we take $r$ to be a very large constant, and $s$ to be a much larger constant, then the above decoding radii $\eta_{r}$ and $\eta_{r^{\prime}}$ approach $\delta=1-R$.

\section{Decoding Multivariate Multiplicity Codes}

\subsection{Local Correction}

We begin by discussing local correction algorithms for multiplicity codes. When coupled with a systematic encoding map (which we discuss in the next section), this also gives local decoding algorithms for multiplicity codes.

\subsubsection{Preliminaries on Restrictions and derivatives}

We first consider the relationship between the derivatives of a multivariate polynomial $P$ and its restrictions to a line. Fix $\mathbf{a}, \mathbf{b} \in \mathbb{F}_{q}^{m}$, and consider the polynomial $Q(T)=P(\mathbf{a}+\mathbf{b} T)$.

- The relationship of $Q(T)$ with the derivatives of $P$ at a: By the definition of Hasse derivatives,

$$
Q(T)=\sum_{\mathbf{i}} P^{(\mathbf{i})}(\mathbf{a}) \mathbf{b}^{\mathbf{i}} T^{\mathrm{wt}(\mathbf{i})}
$$

Grouping terms, we see that:

$$
\sum_{\mathbf{i} \mid \mathbf{w t}(\mathbf{i})=j} P^{(\mathbf{i})}(\mathbf{a}) \mathbf{b}^{\mathbf{i}}=\text { coefficient of } T^{j} \text { in } Q(T) .
$$


- The relationship of the derivatives of $Q$ at $t$ with the derivatives of $P$ at a $+t \mathbf{b}$ : Let $t \in \mathbb{F}_{q}$. By the definition of Hasse derivatives, we get the following two identities:

$$
\begin{gathered}
P(\mathbf{a}+\mathbf{b}(t+R))=Q(t+R)=\sum_{j} Q^{(j)}(t) R^{j} . \\
P(\mathbf{a}+\mathbf{b}(t+R))=\sum_{\mathbf{i}} P^{(\mathbf{i})}(\mathbf{a}+\mathbf{b} t)(\mathbf{b} R)^{\mathbf{i}} .
\end{gathered}
$$

Thus,

$$
Q^{(j)}(t)=\sum_{\mathbf{i} \mid \mathbf{w t}(\mathbf{i})=j} P^{(\mathbf{i})}(\mathbf{a}+\mathbf{b} t) \mathbf{b}^{\mathbf{i}}
$$

In particular, $Q^{(j)}(t)$ is simply a linear combination of the various $P^{(\mathbf{i})}(\mathbf{a}+\mathbf{b} t)$ (over different $\mathbf{i}$ ).

We now apply these observations to the derivatives of $P$. For each nonnegative tuple $\mathbf{e} \in \mathbb{Z}^{m}$, consider the polynomial $Q_{\mathbf{e}}(T)=P^{(\mathbf{e})}(\mathbf{a}+\mathbf{b} T)$.

- The relationship of $Q_{\mathbf{e}}(T)$ with the derivatives of $P$ at a:

$$
\sum_{\mathbf{i} \mid \operatorname{wt}(\mathbf{i})=j}\left(P^{(\mathbf{e})}\right)^{(\mathbf{i})}(\mathbf{a}) \mathbf{b}^{\mathbf{i}}=\sum_{\mathbf{i} \mid \operatorname{wt}(\mathbf{i})=j}\left(\begin{array}{c}
\mathbf{e}+\mathbf{i} \\
\mathbf{e}
\end{array}\right) P^{(\mathbf{e}+\mathbf{i})}(\mathbf{a}) \mathbf{b}^{\mathbf{i}}=\text { coefficient of } T^{j} \text { in } Q_{\mathbf{e}}(T) .
$$

In particular, knowing $Q_{\mathbf{e}}(T)$ gives us several linear relations between the evaluations of the derivatives of $P$ at $\mathbf{a}$.

- The relationship of the derivatives of $Q_{\mathbf{e}}$ at $t$ with the derivatives of $P$ at $\mathbf{a}+t \mathbf{b}$ : Let $t \in \mathbb{F}_{q}$. We get

$$
Q_{\mathbf{e}}^{(j)}(t)=\sum_{\mathbf{i} \mid \mathbf{w t}(\mathbf{i})=j}\left(P^{(\mathbf{e})}\right)^{(\mathbf{i})}(\mathbf{a}+\mathbf{b} t) \mathbf{b}^{\mathbf{i}}=\sum_{\mathbf{i} \mid \mathbf{w t}(\mathbf{i})=j}\left(\begin{array}{c}
\mathbf{e}+\mathbf{i} \\
\mathbf{e}
\end{array}\right) P^{(\mathbf{e}+\mathbf{i})}(\mathbf{a}+\mathbf{b} t) \mathbf{b}^{\mathbf{i}}
$$

In particular, $Q_{\mathbf{e}}^{(j)}(t)$ is simply a linear combination of evaluations, at $\mathbf{a}+\mathbf{b} t$, of the various derivatives of $P$.

\subsubsection{The Local Correction Algorithm}

We now give our local correction algorithm which corrects $\delta_{0}<\frac{\delta}{8}$ fraction errors. The $\gamma=0, c=1$ case of this algorithm is the orignal local correction algorithm of [KSY11. Increasing $\gamma$ reduces the query complexity from $s^{O(m)}$ to $\exp (m)$, while reducing the fraction of correctable errors by a negligible amount.

Main Local Correction Algorithm:

Input: received word $r: \mathbb{F}_{q}^{m} \rightarrow \Sigma$, point $\mathbf{a} \in \mathbb{F}_{q}^{m}$. Abusing notation again, we will write $r^{(\mathbf{i})}(\mathbf{a})$ when we mean the $\mathbf{i}$ coordinate of $r(\mathbf{a})$.

1. Set $\gamma=1-\frac{(1-\delta)}{1-8 \delta_{0}}=\frac{\delta-8 \delta_{0}}{1-8 \delta_{0}}$. Set $c=\gamma \cdot s+1$.

2. Pick a set $B$ of directions: Pick $\mathbf{z}, \mathbf{y}_{1}, \mathbf{y}_{2}, \ldots \mathbf{y}_{m} \in \mathbb{F}_{q}^{m}$ independently and uniformly at random. Let $S \subset \mathbb{F}_{q}$ be any set of size $\left\lceil\frac{5 s}{c}\right\rceil$. Define

$$
B=\left\{\mathbf{z}+\sum_{j=1}^{m} \alpha_{j} \mathbf{y}_{j} \mid \alpha_{j} \in S\right\} .
$$


3. Recover $P^{(\mathbf{e})}(\mathbf{a}+\mathbf{b} T)$ for directions $\mathbf{b} \in B$ : For each $\mathbf{e}$ with $\operatorname{wt}(\mathbf{e})<c$ and each $\mathbf{b} \in B$, consider the function $\ell_{\mathbf{b}, \mathbf{e}}: \mathbb{F}_{q} \rightarrow \mathbb{F}_{q}^{s-\mathrm{wt}(\mathbf{e})}$ given by

$$
\left(\ell_{\mathbf{b}, \mathbf{e}}(t)\right)_{j}=\sum_{\mathbf{i} \mid \mathbf{w t}(\mathbf{i})=j}\left(\begin{array}{c}
\mathbf{e}+\mathbf{i} \\
\mathbf{e}
\end{array}\right) r^{(\mathbf{e}+\mathbf{i})}(\mathbf{a}+\mathbf{b} t) \mathbf{b}^{\mathbf{i}},
$$

for each $0 \leq j<s-$ wt(e). Via a univariate multiplicity code decoding algorithm, find the unique polynomial $Q_{\mathbf{b}, \mathbf{e}}(T) \in \mathbb{F}_{q}[T]$ of degree at most $d-$ wt $(\mathbf{e})$ (if any), such that

$$
\Delta\left(\operatorname{Enc}_{s-\mathrm{wt}(\mathbf{e}), d-\mathrm{wt}(\mathbf{e}), 1, q}\left(Q_{\mathbf{b}, \mathbf{e}}\right), \ell_{\mathbf{b}, \mathbf{e}}\right)<2 \delta_{0} .
$$

4. Decode a constant degree multiplicity code to recover $P^{(<s)}(\mathbf{a})$ : Denote the coefficient of $T^{j}$ in $Q_{\mathbf{b}, \mathbf{e}}(T)$ by $v_{j, \mathbf{b}, \mathbf{e}} \in \mathbb{F}_{q}$. If $j<0$, we define $v_{j, \mathbf{b}, \mathbf{e}}=0$.

For each $j^{\prime}$ with $0 \leq j^{\prime}<s$, find the unique homogeneous degree $j^{\prime}$ polynomial $R_{j^{\prime}}(\mathbf{X}) \in \mathbb{F}_{q}[\mathbf{X}]$ such that for at least $1 / 3$ of the $\mathbf{b} \in B$, for all $\mathbf{e}$ with wt $(\mathbf{e})<c$, we have:

$$
R_{j^{\prime}}^{(\mathbf{e})}(\mathbf{b})=v_{j^{\prime}-\mathrm{wt}(\mathbf{e}), \mathbf{b}, \mathbf{e}} .
$$

Note that this is a constant degree multiplicity code decoding problem.

If such an $R_{j^{\prime}}$ does not exist, or is not unique, the algorithm outputs FAIL.

For each $\mathbf{i}$ with $\mathrm{wt}(\mathbf{i})<s$, define $u_{\mathbf{i}}$ to equal the coefficient of $\mathbf{X}^{\mathbf{i}}$ in $R_{\mathrm{wt}(\mathbf{i})}(\mathbf{X})$.

5. Output the vector $\left\langle u_{\mathbf{i}}\right\rangle_{\mathrm{wt}}(\mathbf{i})<s$.

We quickly comment on the running time and query complexity. The running time consists of $|S|^{m}$ instances of decoding univariate multiplicity codes over $\mathbb{F}_{q}$, as well as on instance of decoding a degree- $s \mathrm{~m}$-variate order-c multivariate multiplicity code with evaluation points being $S^{m}$. Thus, if $m, s$ are constant, the running time is near-linear in $q$, which is near-linear in $n^{1 / m}$, where $n$ is the block-length of the code. The query complexity is $|S|^{m} \cdot q$, which equals $\left(\frac{5}{\gamma}\right)^{m} \cdot n^{1 / m}$. For $\delta=\Omega(1)$ and $\delta_{0}<\delta / 10$ (say), the query complexity equals $\exp (m) \cdot n^{1 / m}$.

\subsubsection{Analysis of the Local Correction Algorithm}

We now analyze the above local correction algorithm.

Theorem 7 Let $P(\mathbf{X}) \in \mathbb{F}_{q}[\mathbf{X}]$ be such that $\Delta\left(\operatorname{Enc}_{s, d, m, q}(P), r\right)<\delta_{0}$. Let $\mathbf{a} \in \mathbb{F}_{q}^{m}$.

With high probability, the local correction algorithm above outputs $P^{(<s)}(\mathbf{a})$.

Proof Let $E=\left\{x \in \mathbb{F}_{q}^{m} \mid P^{(<s)}(x) \neq r^{(<s)}(x)\right\}$ be the error set. We have $|E|<\delta_{0} \cdot q^{m}$.

Let $L_{\mathbf{b}}=\left\{\mathbf{a}+t \mathbf{b} \mid t \in \mathbb{F}_{q}\right\}$ be the line through $\mathbf{a}$ in direction $\mathbf{b}$. We call $\mathbf{b}$ bad if $\left|L_{\mathbf{b}} \cap E\right| \geq 4 \cdot \delta_{0} \cdot q$.

Note that at most $1 / 4$ of all the lines are bad.

Claim 8 With high probability, we have:

1. at most $1 / 3$ of the $\mathbf{b} \in B$ are bad,

2. $|B|=|S|^{m}$, 
These basic probability/linear-algebra facts are well known, and we omit the proofs.

Henceforth we assume that both these events happen.

Claim 9 If $\mathbf{b}$ is good, then for every $\mathbf{e}$ with $\mathrm{wt}(\mathbf{e})<c$, we have:

$$
Q_{\mathbf{b}, \mathbf{e}}(T)=P^{(\mathbf{e})}(\mathbf{a}+\mathbf{b} T)
$$

Proof The univariate multiplicity code of order $s-\operatorname{wt}(\mathbf{e})$ evaluations of degree $d-$ wt $(\mathbf{e})$ polynomials has minimum distance at least $1-\frac{d}{(s-c+1) q}=1-\frac{1-\delta}{1-\gamma}$ which, by choice of $\gamma$, is $\geq 8 \cdot \delta_{0}$.

If $\mathbf{b}$ is good, then we know that $\left|L_{\mathbf{b}} \cap E\right|<4 \cdot \delta_{0} \cdot q$. By Equations (7) and (6), we conclude that $P^{(\mathbf{e})}(\mathbf{a}+\mathbf{b} T)$ (which has degree $d-\operatorname{wt}(\mathbf{e}))$ satisfies:

$$
\Delta\left(\operatorname{Enc}_{s-\mathrm{wt}(\mathbf{e}), d-\mathrm{wt}(\mathbf{e}), 1, q}\left(P^{(\mathbf{e})}(\mathbf{a}+\mathbf{b} T)\right), \ell_{\mathbf{b}, \mathbf{e}}\right) \leq \frac{\left|L_{\mathbf{b}} \cap E\right|}{q}<4 \cdot \delta_{0},
$$

which is less than half the minimum distance of the univariate multiplicity code of order $s-$ wt $(\mathbf{e})$ evaluations of degree $d-\operatorname{wt}(\mathbf{e})$ polynomials.

Thus $P^{(\mathbf{e})}(\mathbf{a}+\mathbf{b} T)$ is the unique such polynomial found in Step 3, and so $Q_{\mathbf{b}, \mathbf{e}}(T)=P^{(\mathbf{e})}(\mathbf{a}+\mathbf{b} T)$.

For each integer $0 \leq j^{\prime}<s$, define the polynomial:

$$
\tilde{R}_{j^{\prime}}(\mathbf{X})=\sum_{\mathbf{i}^{\prime} \mid \operatorname{wt}\left(\mathbf{i}^{\prime}\right)=j^{\prime}} P^{\left(\mathbf{i}^{\prime}\right)}(\mathbf{a}) \mathbf{X}^{\mathbf{i}^{\prime}}
$$

Claim 10 If $\mathbf{b}$ is good, then for all $\mathbf{e}$ with $\mathrm{wt}(\mathbf{e})<c$, we have:

$$
\tilde{R}_{j^{\prime}}^{(\mathbf{e})}(\mathbf{b})=v_{j^{\prime}-\mathrm{wt}(\mathbf{e}), \mathbf{b}, \mathbf{e}}
$$

Proof We have:

$$
\begin{aligned}
\tilde{R}_{j^{\prime}}^{(\mathbf{e})}(\mathbf{X}) & =\sum_{\mathbf{i}^{\prime} \mid \operatorname{wt}\left(\mathbf{i}^{\prime}\right)=j^{\prime}}\left(\begin{array}{c}
\mathbf{i}^{\prime} \\
\mathbf{e}
\end{array}\right) P^{\left(\mathbf{i}^{\prime}\right)}(\mathbf{a}) \mathbf{X}^{\mathbf{i}^{\prime}-\mathbf{e}} \\
& =\sum_{\mathbf{i}^{\prime} \mid \operatorname{wt}\left(\mathbf{i}^{\prime}\right)=j^{\prime}, \mathbf{i}^{\prime} \geq \mathbf{e}}\left(\begin{array}{c}
\mathbf{i}^{\prime} \\
\mathbf{e}
\end{array}\right) P^{\left(\mathbf{i}^{\prime}\right)}(\mathbf{a}) \mathbf{X}^{\mathbf{i}^{\prime}-\mathbf{e}} \\
& =\sum_{\mathbf{i} \mid \operatorname{wt}(\mathbf{i})=j}\left(\begin{array}{c}
\mathbf{e}+\mathbf{i} \\
\mathbf{e}
\end{array}\right) P^{(\mathbf{e}+\mathbf{i})}(\mathbf{a}) \mathbf{X}^{\mathbf{i}},
\end{aligned}
$$

where $j=j^{\prime}-\operatorname{wt}(\mathbf{e})$.

Thus,

$$
\begin{aligned}
\tilde{R}_{j^{\prime}}^{(\mathbf{e})}(\mathbf{b}) & =\sum_{\mathbf{i} \mid \operatorname{wt}(\mathbf{i})=j}\left(\begin{array}{c}
\mathbf{e}+\mathbf{i} \\
\mathbf{e}
\end{array}\right) P^{(\mathbf{e}+\mathbf{i})}(\mathbf{a}) \mathbf{b}^{\mathbf{i}} \\
& =\text { coeff. of } T^{j} \text { in } Q_{\mathbf{b}, \mathbf{e}}(T) \quad \text { (by Equation (5) and Claim 9, since } \mathbf{b} \text { is good) } \\
& =v_{j^{\prime}-\operatorname{wt}(\mathbf{e}), \mathbf{b}, \mathbf{e} .}
\end{aligned}
$$


Thus $\tilde{R}_{j^{\prime}}(\mathbf{X})$ satisfies the conditions required of Step 4 of the algorithm.

Let us now show that no other polynomial can satisfy these conditions. Suppose there was some other solution $\bar{R}_{j^{\prime}}(\mathbf{X})$. Then the difference $\left(\tilde{R}_{j^{\prime}}-\bar{R}_{j^{\prime}}\right)(\mathbf{X})$ would be a nonzero polynomial of degree $<s$, that vanishes with multiplicity at least $c$, at $\geq \frac{1}{3}$ of the points of $B$. But this cannot be, since $B$ is an affine one-to-one image of the set $S^{m}$, and the fraction of points of $S^{m}$ on which a nonzero polynomial of degree $<s$ can vanish with multiplicity $\geq c$ is at most $\frac{s}{c|S|}=\frac{1}{5}<\frac{1}{3}$. Thus $\tilde{R}_{j^{\prime}}$ is the unique solution found in Step 4 .

Finally, we notice that our definition of $R_{j^{\prime}}$ implies that for every $\mathbf{i}$, we have $u_{\mathbf{i}}=P^{(\mathbf{i})}(\mathbf{a})$, as desired.

\subsubsection{Variations}

The above algorithm allows a number of variations that may be useful in different contexts.

Let $\mathbf{a} \in \mathbb{F}_{q}^{m}$. Suppose $r: \mathbb{F}_{q}^{m} \rightarrow \Sigma$ is a received word, and suppose $P(\mathbf{X}) \in \mathbb{F}_{q}[\mathbf{X}]$ is a polynomial of degree at most $d$ such that $\Delta\left(\operatorname{Enc}_{s, d, m, q}(P), r\right)<\delta_{0}$. Let $\gamma=\frac{\delta-8 \delta_{0}}{1-8 \delta_{0}}$, and let $c=\gamma s+1$.

Let $\mathbf{a} \in \mathbb{F}_{q}^{m}$. For each integer $0 \leq j^{\prime}<s$, define the polynomial:

$$
\tilde{R}_{j^{\prime}}(\mathbf{X})=\sum_{\mathbf{i}^{\prime} \mid \operatorname{wt}\left(\mathbf{i}^{\prime}\right)=j^{\prime}} P^{\left(\mathbf{i}^{\prime}\right)}(\mathbf{a}) \mathbf{X}^{\mathbf{i}^{\prime}}
$$

Suppose $\mathbf{b} \in \mathbb{F}_{q}^{m}$ is good (meaning that the line $L_{\mathbf{a}, \mathbf{b}}$ has $<4 \delta_{0} q$ errors on it). As we saw in the above analysis, by querying all the points of the line $L_{\mathbf{a}, \mathbf{b}}$, we can compute $\tilde{R}_{j^{\prime}}^{(\mathbf{e})}(\mathbf{b})$, for every $j^{\prime}<s$ and every $\mathbf{e}$ such that wt $(\mathbf{e})<c$.

1. Suppose we are only interested in recovering $P^{(<c)}(\mathbf{a})$. Then this can be recovered by querying the points of just one line! Indeed, if we pick $\mathbf{b}$ at random, then with high probability $\mathbf{b}$ is good, and then by querying $L_{\mathbf{a}, \mathbf{b}}$ we can compute $\tilde{R}_{j^{\prime}}^{(\mathbf{e})}(\mathbf{b})$ for every $j^{\prime}<s$ and every $\mathbf{e}$ such that $\operatorname{wt}(\mathbf{e})<c$. Note that for every e with $\mathrm{wt}(\mathbf{e})<c$, we have $\tilde{R}_{\mathrm{wt}(\mathbf{e})}^{(\mathbf{e})}(\mathbf{b})=P^{(\mathbf{e})}(\mathbf{b})$. Thus we can compute $P^{(<c)}(\mathbf{a})$ with high probability.

We now describe a coding scheme taking advantage of this. Specializing parameters, if we take $\delta=1 / 2$, $\delta_{0}=1 / 100, s=\Omega\left(m^{2}\right), d=(1-\delta) \cdot s \cdot q, n=q^{m}, \gamma=\frac{1}{5}$, and so $c>\frac{s}{5}$. Let $\Sigma_{0}=\mathbb{F}_{q}^{\{\mathbf{i} \mid \operatorname{wt}(\mathbf{i})<c\}}$, and let our space of messages be the space of all functions $f: \mathbb{F}_{q}^{m} \rightarrow \Sigma_{0}$.

Define the encoding of the message $f$ as follows: find any polynomial $P(\mathbf{X}) \in \mathbb{F}_{q}[\mathbf{X}]$ of degree at most $(c+m) \cdot q$ such that for each $\mathbf{a} \in \mathbb{F}_{q}^{m}$, we have $P^{(<c)}(\mathbf{a})=f(\mathbf{a})$ (that such a polynomial $P$ exists is an interpolatability statement; it follows from the arguments in Appendix A of Kop12). By choice of parameters, $(c+m) \cdot q<d$. The encoding of $f$ is then defined to be $\operatorname{Enc}_{s, d, m, q}(P) \in \Sigma^{n}$. Note that $\log |\Sigma|=\left(\begin{array}{c}m+s-1 \\ m\end{array}\right) \cdot \log q$, and $\log \left|\Sigma_{0}\right|=\left(\begin{array}{c}m+c-1 \\ m\end{array}\right) \cdot \log q \approx \gamma^{m} \cdot \log |\Sigma|$. Thus this encoding blows up the bit-length of an alphabet symbol by a factor of $5^{m}$, which is at most a sublinear polynomial in $n$ for $q>5$.

By the earlier discussion on local correction, this coding scheme has the following interesting property: given any $\mathbf{a} \in \mathbb{F}_{q}^{m}$, given oracle access to some $r \in \Sigma^{m}$ which is $\delta_{0}$-close to the encoding of $f$, one can recover the value of $f(\mathbf{a})$ with high probability using only $q$ queries into $r$. This scheme can be used even for $q$ as small as $O(1)$ !

2. We now describe another local correction algorithm for multiplicity codes. This algorithm queries only $m$ lines, but it only works for multiplicity codes of low rate. 
To locally correct the value of $P^{(<s)}(\mathbf{a})$ for $\mathbf{a} \in \mathbb{F}_{q}^{m}$, given oracle access to $r: \mathbb{F}_{q}^{m} \rightarrow \Sigma$, the algorithm works as follows. First pick a set $B$ of $m$ uniformly random directions from $\mathbb{F}_{q}^{m}$. With high probability, we will have that $B$ will be a set of $m$ linearly independent vectors, and if the fraction of errors $\delta_{0}$ is sufficiently small, then all the $\mathbf{b} \in B$ will be good. This means that we can compute $\tilde{R}_{j^{\prime}}^{(\mathbf{e})}$ (b) for each $\mathbf{b} \in B, j^{\prime}<s$ and $\mathbf{e}$ with wt $(\mathbf{e})<c$.

The following lemma implies that this data uniquely determines the polynomial $\tilde{R}_{j^{\prime}}(\mathbf{X})$ for each $j^{\prime}<$ $c^{\prime}=c \cdot \frac{m}{m-1}$.

Lemma 11 Let $c^{\prime}=c \cdot \frac{m}{m-1}$. Let $R\left(X_{1}, \ldots, X_{m}\right)$ be a homogeneous polynomial of degree $j^{\prime}<c^{\prime}$. Suppose $B$ is a set of $m$ linearly independent vectors in $\mathbb{F}_{q}^{m}$, such that $\operatorname{mult}(R, \mathbf{b}) \geq c$ for each $\mathbf{b} \in B$. Then $R\left(X_{1}, \ldots, X_{m}\right)=0$.

Proof Without loss of generality, we may assume $B=\left\{\mathbf{u}_{1}, \ldots, \mathbf{u}_{m}\right\}$, where $\mathbf{u}_{i} \in \mathbb{F}_{q}^{m}$ is 1 in coordinate $i$ and 0 in every other coordinate. The hypothesis $\operatorname{mult}\left(R, \mathbf{u}_{i}\right) \geq c$ implies that for every $\mathbf{i}$ with wt $(\mathbf{i})=j^{\prime}$ and $\mathbf{i}_{i}>j^{\prime}-c$, the coefficient of $\mathbf{X}^{\mathbf{i}}$ in $R(\mathbf{X})$ is 0 .

Finally, notice that for every $\mathbf{i}$ with $\mathrm{wt}(\mathbf{i})=j^{\prime}$, there always exists some $i \in[m]$ for which $\mathbf{i}_{i}>\frac{j^{\prime}}{m}>j^{\prime}-c$. Thus $R(\mathbf{X})=0$.

Once we have computed $\tilde{R}_{j^{\prime}}(\mathbf{X})$ for each $j^{\prime}<c^{\prime}=c \cdot \frac{m}{m-1}$, this immediately gives us $P^{\left(<c^{\prime}\right)}(\mathbf{a})$. If $c^{\prime} \geq s$, then this is proper local correction algorithm, but even if $c^{\prime}<s$ this algorithm could be of interest.

For completeness, we record the basic multiplicity amplification fact underlying the above decoding algorithm. This can also be proved using Lemma 11

Lemma 12 Let $c^{\prime}=c \cdot \frac{m}{m-1}$.

Let $\mathbf{a} \in \mathbb{F}^{m}$. Let $P\left(X_{1}, \ldots, X_{n}\right) \in \mathbb{F}\left[X_{1}, \ldots, X_{m}\right]$, and let $B \subseteq \mathbb{F}_{q}^{m}$ be a basis for $\mathbb{F}_{q}^{m}$ over $\mathbb{F}_{q}$. For each $\mathbf{b} \in B$ and each $m$-tuple $\mathbf{e}$ of nonnegative integers, define $Q_{\mathbf{b}, \mathbf{e}}(T)=P^{(\mathbf{e})}(\mathbf{a}+\mathbf{b} T) \in \mathbb{F}[T]$.

Suppose $Q_{\mathbf{b}, \mathbf{e}}(T)=0$ for each $\mathbf{b} \in B$ and each $\mathbf{e}$ with $\operatorname{wt}(\mathbf{e})<c$. Then $\operatorname{mult}(P, \mathbf{a}) \geq c^{\prime}$.

\subsection{Global Decoding}

We now consider decoding of multivariate multiplicity codes in the global sense. In the case of standard polynomial codes (the $s=1$ case), the best known algorithm (due to Pellikaan-Wu [PW04]) for decoding $m$-variate codes over $\mathbb{F}_{q}$ works via a reduction to the decoding of 1-variate codes over the bigger field $\mathbb{F}_{q^{m}}$.

For multiplicity codes with general $s$, Kopparty Kop12 gave a reduction from $m$-variate codes over $\mathbb{F}_{q}$ to several instances of decoding a 1-variate code over the big field $\mathbb{F}_{q^{m}}$. Using the algorithms for decoding univariate multiplicity codes discussed earlier, this gives polynomial time algorithms for unique decoding multivariate multiplicity codes upto half the minimum distance, and list-decoding multivariate multiplicity codes upto the Johnson bound.

Below we describe a variation of the reduction of Kop12. The key ingredient of that reduction is the construction of a certain special family of "algebraically-repelling" curves.

Abusing notation, we call $\mathfrak{a} \in \mathbb{F}_{q^{m}}^{m}$ a basis if its $m$ coordinates form a basis for $\mathbb{F}_{q^{m}}$ over $\mathbb{F}_{q}$. To every basis $\mathfrak{a}=\left(a_{1}, \ldots, a_{m}\right)$, we associate a curve $\gamma_{\mathfrak{a}}(T) \in \mathbb{F}_{q^{m}}[T]^{m}$, given by:

$$
\gamma_{\mathfrak{a}}(T)=\left(\operatorname{Tr}\left(a_{1} T\right), \operatorname{Tr}\left(a_{2} T\right), \ldots, \operatorname{Tr}\left(a_{m} T\right)\right) .
$$


The most interesting feature of this curve is that $\gamma_{\mathfrak{a}}$ is a bijection between $\mathbb{F}_{q^{m}}$ and $\mathbb{F}_{q}^{m}$. See Kop12 for more properties of these curves $\gamma_{\mathfrak{a}}$.

A collection $\mathfrak{a}_{1}, \ldots, \mathfrak{a}_{M} \in \mathbb{F}_{q^{m}}^{m}$ of bases is said to be in $(s, c)$-general position if there does not exist a nonzero polynomial $R(\mathbf{X}) \in \mathbb{F}_{q^{m}}[\mathbf{X}]$ of degree at most $s$ which vanishes at each $\mathfrak{a}_{i}$ with multiplicity at least $c$.

The $c=1$ case of the following lemma was shown in Kop12.

Lemma 13 Suppose $c \leq s<q$. Let $\mathfrak{a}_{1}, \ldots, \mathfrak{a}_{M} \in \mathbb{F}_{q^{m}}^{m}$ be bases in $(s, c)$ general position. Let $Q(\mathbf{X}) \in \mathbb{F}_{q^{m}}[\mathbf{X}]$ have degree $<s \cdot q$. Suppose that for each $i \in[M]$ and each $\mathbf{e}$ with $\operatorname{wt}(\mathbf{e})<c$, the univariate polynomial $Q^{(\mathbf{e})} \circ \gamma_{\mathfrak{a}_{i}}(T)=0$.

Then $Q(\mathbf{X})=0$.

The proof of this lemma is postponed to Section 4.2 .2 .

Explicit collections $\mathfrak{a}_{1}, \ldots, \mathfrak{a}_{M} \in \mathbb{F}_{q^{m}}^{m}$ in $(s, c)$ general position with $M=\left(\frac{s}{c}\right)^{m}$ can be constructed as follows. Take $\mathfrak{a}$ to be a basis of $\mathbb{F}_{q^{m}}^{m}$, and $\mathbf{b}_{1}, \ldots, \mathbf{b}_{M}$ to be all the elements of a $m$-dimensional grid of side $\frac{s}{c}$ in $\mathbb{F}_{q}^{m}$, and set $\mathfrak{a}_{i}=\mathfrak{a}+\mathbf{b}_{i}$.

Via the lemma, this gives us an explicit collection of $\left(\frac{s}{c}\right)^{m}$ "algebraically-repelling" curves. We now show how these curves can be used for reducing multivariate decoding to univariate decoding. Again, this generalizes the $\gamma=0, c=1$ case, which was done in Kop12.

The following lemma, relating the derivatives of a multivariate polynomial to the derivatives of its restriction to the curve $\gamma_{\mathfrak{a}}$, will motivate one of the steps of the algorithm.

Lemma 14 Let $P\left(X_{1}, \ldots, X_{m}\right) \in \mathbb{F}_{q}\left[X_{1}, \ldots, X_{m}\right]$ and let $Q_{\mathbf{e}}(T) \in \mathbb{F}_{q^{m}}[T]$ be given by $Q_{\mathbf{e}}(T)=P^{(\mathbf{e})} \circ \gamma_{\mathfrak{a}}(T)$. Then for every $t \in \mathbb{F}_{q^{m}}$ and every $j<q$ :

$$
Q_{\mathbf{e}}^{(j)}(t)=\sum_{\mathbf{i}: \mathrm{wt}(\mathbf{i})=j}\left(\begin{array}{c}
\mathbf{i}+\mathbf{e} \\
\mathbf{i}
\end{array}\right) P^{(\mathbf{i}+\mathbf{e})}\left(\gamma_{\mathfrak{a}}(t)\right) \mathfrak{a}^{\mathbf{i}}
$$

\section{Algorithm for Reducing Multivariate Decoding to Univariate Decoding}

1. Suppose we have an algorithm $\mathcal{A}$ that list-decodes univariate multiplicity codes of distance $\delta$ from $\eta(\delta)$-fraction errors.

2. Let $\gamma=\frac{\delta-\eta^{-1}\left(\delta_{0}\right)}{1-\eta^{-1}\left(\delta_{0}\right)}$, and let $c=\gamma \cdot s+1$.

3. Let $M=\left(\frac{s}{c}\right)^{m}$. Pick bases $\mathfrak{a}_{1}, \mathfrak{a}_{2}, \ldots, \mathfrak{a}_{M} \in \mathbb{F}_{q^{m}}^{m}$ in $(s, c)$-general position.

4. For each $i \in[M]$, for each $\mathbf{e}$ with wt $(\mathbf{e})<c$, define $\ell_{i, \mathbf{e}}: \mathbb{F}_{q^{m}} \rightarrow \mathbb{F}_{q^{m}}^{s-c+1}$ as follows. For each $j$ with $0 \leq j<s-c+1$, let

$$
\left(\ell_{i, \mathbf{e}}(t)\right)_{j}=\sum_{\mathbf{i}: \mathrm{wt}(\mathbf{i})=j}\left(\begin{array}{c}
\mathbf{i}+\mathbf{e} \\
\mathbf{e}
\end{array}\right) r^{(\mathbf{i}+\mathbf{e})}\left(\gamma_{\mathfrak{a}_{i}}(t)\right) \cdot \mathfrak{a}_{i}^{\mathbf{i}} .
$$

5. Using algorithm $\mathcal{A}$, compute the set $\mathcal{L}_{i, \mathbf{e}}$ of all $Q(T) \in \mathbb{F}_{q^{m}}[T]$ of degree at most $d q^{m-1}$ such that $\Delta\left(\operatorname{Enc}_{s-c+1, d q^{m-1}, 1, q^{m}}(Q), \ell_{i, \mathbf{e}}\right)<\delta_{0}$.

6. For every $\left(Q_{1}(T), Q_{2}(T), \ldots, Q_{M}(T)\right) \in \prod_{i=1}^{M} \mathcal{L}_{i}$, find all $P\left(X_{1}, \ldots, X_{m}\right) \in \mathbb{F}_{q}\left[X_{1}, \ldots, X_{m}\right]$ with $\operatorname{deg}(P) \leq d$ such that for each $i \in[M]$,

$$
P \circ \gamma_{\mathfrak{a}_{i}}(T)=Q_{i}(T) .
$$

(This is a system of linear equations over $\mathbb{F}_{q^{m}}$ with $\left(\begin{array}{c}d+m \\ m\end{array}\right)$ variables and $(d+1) \cdot M$ constraints). 
7. Output the list of all such $P\left(X_{1}, \ldots, X_{m}\right)$.

Before analyzing correctness, let us comment on the running time of this reduction. For constant $m, s$, we claim that this reduction can be implemented to run in near-linear time. The running time of this reduction depends the size $\mathcal{L}_{i}$, and on how quickly we can find $P\left(X_{1}, \ldots, X_{m}\right)$ given $Q_{1}(T), \ldots, Q_{M}(T)$. If we happen to know that the $\mathcal{L}_{i}$ are all of constant size (as it happens whenever the fraction of errors is bounded below the Johnson radius), then the factor of $\prod\left|\mathcal{L}_{i}\right|$ is at most a constant. Thus we only need to show that the latter step can be implemented in near-linear time. Given $Q_{1}(T), \ldots, Q_{M}(T)$, we can: (1) evaluate each $Q_{i}(T)$ and each of its derivatives of order $<s$ at all points of $\mathbb{F}_{q}^{m},(2)$ via Lemma 14, we know that this suffices to give us $P^{(<s)}(\mathbf{a})$ for each $\mathbf{a} \in \mathbb{F}_{q}^{m},(3)$ to interpolate $P(\mathbf{X})$ given $P^{(<s)}(\mathbf{a})$ for each $\mathbf{a} \in \mathbb{F}_{q}^{m}$.

Thus, we get that unique decoding of multivariate multiplicity codes upto half the minimum distance can be done in near-linear time. Furthermore, if Nielsen's algorithm for list-decoding univariate multiplicity codes upto the Johnson bound can be implemented in near-linear time, then one can also list-decode multivariate multiplicity codes upto (almost) the Johnson bound in near-linear time.

\subsubsection{Analysis of the decoding algorithm}

Suppose $P(\mathbf{X}) \in \mathbb{F}_{q}[\mathbf{X}]$ is such that $\Delta\left(\operatorname{Enc}_{s, d, m, q}(P), r\right)<\delta_{0}$. Let $E \subseteq \mathbb{F}_{q}^{m}$ be the set of a $\in \mathbb{F}_{q}^{m}$ where $P^{(<s)}(\mathbf{a})$ differs from $\left.r^{(<s}\right)(\mathbf{a})$.

We first show that $Q_{i, \mathbf{e}}(T):=P^{(\mathbf{e})} \circ \gamma_{\mathfrak{a}_{i}}(T) \in \mathcal{L}_{i, \mathbf{e}}$. Indeed, by Lemma 14 for every $t \in \mathbb{F}_{q^{m}}$ such that $\gamma_{\mathfrak{a}}(t) \notin E$, and for every $j<s-c+1$, we have:

$$
\begin{aligned}
Q_{i, \mathbf{e}}^{(j)}(t) & =\sum_{\mathbf{i}: \mathrm{wt}(\mathbf{i})=j}\left(\begin{array}{c}
\mathbf{i}+\mathbf{e} \\
\mathbf{i}
\end{array}\right) P^{(\mathbf{i}+\mathbf{e})}\left(\gamma_{\mathfrak{a}_{i}}(t)\right) \mathfrak{a}_{i}^{\mathbf{i}} \\
& =\sum_{\mathbf{i}: \mathrm{wt}(\mathbf{i})=j}\left(\begin{array}{c}
\mathbf{i}+\mathbf{e} \\
\mathbf{i}
\end{array}\right) r^{(\mathbf{i}+\mathbf{e})}\left(\gamma_{\mathfrak{a}_{i}}(t)\right) \mathfrak{a}_{i}^{\mathbf{i}} \\
& =\sum_{\mathbf{i}: \mathbf{w t}(\mathbf{i})=j}\left(\begin{array}{c}
\mathbf{i}+\mathbf{e} \\
\mathbf{i}
\end{array}\right) r^{(\mathbf{i}+\mathbf{e})}\left(\gamma_{\mathfrak{a}_{i}}(t)\right) \mathfrak{a}_{i}^{\mathbf{i}} \\
& =\ell_{i, \mathbf{e}}(t) .
\end{aligned}
$$

Thus $\Delta\left(\operatorname{Enc}_{s-c+1, d, 1, q}\left(Q_{i, \mathbf{e}}\right), \ell_{i, \mathbf{e}}\right) \leq \frac{|E|}{q^{m}}<\delta_{0}$, and thus $Q_{i, \mathbf{e}}$ is indeed included in the list $\mathcal{L}_{i}$. The crucial points here are (a) the relative distance of the univariate multiplicity code of order $s-c+1$ evaluations of degree $d$ polynomials has minimum distance $1-\frac{1-\delta}{1-\gamma}$, and (b) our choice of $\gamma$ ensures that $\delta_{0} \leq \eta\left(1-\frac{1-\delta}{1-\gamma}\right)$. Thus algorithm $\mathcal{A}$ is indeed capable of finding $\mathcal{L}_{i}$ as required by the reduction.

\subsubsection{Algebraically repelling curves}

In this section we prove Lemma 14 and Lemma 13

Proof of Lemma 14; By definition of derivatives, we have:

$$
\begin{gathered}
Q_{\mathbf{e}}(t+W)=\sum_{j} Q_{\mathbf{e}}^{(j)}(t) W^{j} \\
P^{(\mathbf{e})}\left(\gamma_{\mathfrak{a}}(t)+\mathbf{X}\right)=\sum_{\mathbf{i}}\left(P^{(\mathbf{e})}\right)^{(\mathbf{i})}\left(\gamma_{\mathfrak{a}}(t)\right) \mathbf{X}^{\mathbf{i}}=\sum_{\mathbf{i}}\left(\begin{array}{c}
\mathbf{e}+\mathbf{i} \\
\mathbf{i}
\end{array}\right) P^{(\mathbf{e}+\mathbf{i})}\left(\gamma_{\mathfrak{a}}(t)\right) \mathbf{X}^{\mathbf{i}}
\end{gathered}
$$


By linearity, $\gamma_{\mathfrak{a}}(t+W)=\gamma_{\mathfrak{a}}(t)+\gamma_{\mathfrak{a}}(W)$. So

$$
\begin{aligned}
Q_{\mathbf{e}}(t+W) & =P^{(\mathbf{e})} \circ \gamma_{\mathfrak{a}}(t+W) \\
& =P^{(\mathbf{e})}\left(\gamma_{\mathfrak{a}}(t)+\gamma_{\mathfrak{a}}(W)\right) \\
& =\sum_{\mathbf{i}}\left(\begin{array}{c}
\mathbf{e}+\mathbf{i} \\
\mathbf{i}
\end{array}\right) P^{(\mathbf{e}+\mathbf{i})}\left(\gamma_{\mathfrak{a}}(t)\right)\left(\gamma_{\mathfrak{a}}(W)\right)^{\mathbf{i}}
\end{aligned}
$$

Taking this equation $\bmod W^{q}$, we get the following equation:

$$
\sum_{j<q} Q_{\mathbf{e}}^{(j)}(t) W^{j}=\sum_{\mathbf{i}: \mathbf{w t}(\mathbf{i})<q}\left(\begin{array}{c}
\mathbf{e}+\mathbf{i} \\
\mathbf{i}
\end{array}\right) P^{(\mathbf{e}+\mathbf{i})}\left(\gamma_{\mathfrak{a}}(t)\right)(\mathfrak{a} W)^{\mathbf{i}} \quad \bmod W^{q}
$$

For $j<q$, note that the coefficient of $W^{j}$ in the right hand side of this equation equals $\sum_{\mathbf{i}: \operatorname{wt}(\mathbf{i})=j}\left(\begin{array}{c}\mathbf{e}+\mathbf{i} \\ \mathbf{i}\end{array}\right) P^{(\mathbf{e}+\mathbf{i})}\left(\gamma_{\mathfrak{a}}(t)\right) \mathfrak{a}^{\mathbf{i}}$. On the other hand, the coefficient of $W^{j}$ in the left hand side of the equation equals $Q_{\mathbf{e}}^{(j)}(t)$. We therefore conclude that for each $j$ with $0 \leq j<q$, we have

$$
Q_{\mathbf{e}}^{(j)}(t)=\sum_{\mathbf{i}: \mathbf{w t}(\mathbf{i})=j}\left(\begin{array}{c}
\mathbf{e}+\mathbf{i} \\
\mathbf{i}
\end{array}\right) P^{(\mathbf{e}+\mathbf{i})}\left(\gamma_{\mathfrak{a}}(t)\right) \mathfrak{a}^{\mathbf{i}}
$$

Proof of Lemma 13: We will show that for each $\mathbf{a} \in \mathbb{F}_{q}^{m}, \operatorname{mult}(P, \mathbf{a}) \geq s$. Then by Lemma 4 (recalling that $\operatorname{deg}(P)<s q)$, we can conclude that $P\left(X_{1}, \ldots, X_{m}\right)=0$.

Fix $i \in[M]$ and $\mathbf{e}$ with $\operatorname{wt}(\mathbf{e})<c$. We have $P^{(\mathbf{e})} \circ \gamma_{\mathfrak{a}_{i}}(T)=0$. By Lemma 14, we conclude that for every $t \in \mathbb{F}_{q^{m}}$ and $j<q$ :

$$
\sum_{\mathbf{i}: \mathrm{wt}(\mathbf{i})=j}\left(\begin{array}{c}
\mathbf{e}+\mathbf{i} \\
\mathbf{i}
\end{array}\right) P^{(\mathbf{e}+\mathbf{i})}\left(\gamma_{\mathfrak{a}_{i}}(t)\right) \mathfrak{a}_{i}^{\mathbf{i}}=0 .
$$

Thus for every $\mathbf{a} \in \mathbb{F}_{q}^{m}$, every $i \in[M], 0 \leq j<s$, and $\mathbf{e}$ with $\operatorname{wt}(\mathbf{e})<c$ :

$$
\sum_{\mathbf{i}: \operatorname{wt}(\mathbf{i})=j}\left(\begin{array}{c}
\mathbf{e}+\mathbf{i} \\
\mathbf{i}
\end{array}\right) P^{(\mathbf{e}+\mathbf{i})}(\mathbf{a}) \mathfrak{a}_{i}^{\mathbf{i}}=0 .
$$

For $0 \leq j^{\prime}<q$, let $R_{\mathbf{a}, j^{\prime}}(\mathbf{Y}) \in \mathbb{F}_{q}[\mathbf{Y}]$ be the polynomial

$$
R_{\mathbf{a}, j^{\prime}}(\mathbf{Y})=\sum_{\mathbf{i}^{\prime}: \operatorname{wt}\left(\mathbf{i}^{\prime}\right)=j^{\prime}} P^{\left(\mathbf{i}^{\prime}\right)}(\mathbf{a}) \mathbf{Y}^{\mathbf{i}^{\prime}}
$$

Then the derivatives of $R_{\mathbf{a}, j^{\prime}}$ are given by:

$$
\begin{aligned}
R_{\mathbf{a}, j^{\prime}}^{(\mathbf{e})}(\mathbf{Y}) & =\sum_{\mathbf{i}^{\prime}: \operatorname{wt}\left(\mathbf{i}^{\prime}\right)=j^{\prime}}\left(\begin{array}{c}
\mathbf{i}^{\prime} \\
\mathbf{e}
\end{array}\right) P^{\left(\mathbf{i}^{\prime}\right)}(\mathbf{a}) \mathbf{Y}^{\mathbf{i}^{\prime}-\mathbf{e}} \\
& =\sum_{\mathbf{i}: \operatorname{wt}(\mathbf{i})=j^{\prime}-\operatorname{wt}(\mathbf{e})}\left(\begin{array}{c}
\mathbf{i}+\mathbf{e} \\
\mathbf{e}
\end{array}\right) P^{(\mathbf{i}+\mathbf{e})}(\mathbf{a}) \mathbf{Y}^{\mathbf{i}}
\end{aligned}
$$


Equation (8) says that for each $\mathbf{a} \in \mathbb{F}_{q}^{m}, i \in[M]$, e with wt $(\mathbf{e})<c$, and $j^{\prime}<q+\mathrm{wt}(\mathbf{e})$, we have

$$
R_{\mathbf{a}, j^{\prime}}^{(\mathbf{e})}\left(\mathfrak{a}_{i}\right)=0 .
$$

Thus for every $j<q$ and $\mathbf{a}, i$, e as above:

$$
\operatorname{mult}\left(R_{\mathbf{a}, j}, \mathfrak{a}_{i}\right) \geq c .
$$

By the general position hypothesis on $\mathfrak{a}_{i}$, this implies that for each $j<s$, the polynomial $R_{\mathbf{a}, j}(\mathbf{Y})$ is itself identically 0 .

But the coefficients of $R_{\mathbf{a}, j}(\mathbf{Y})$ are $P^{(\mathbf{i})}(\mathbf{a})$, for $\mathbf{i}$ satisfying wt(i) $=j$. Thus $P^{(\mathbf{i})}(\mathbf{a})=0$ for each $\mathbf{a}$ and each i with wt $(\mathbf{i})<s$.

Therefore $\operatorname{mult}(P, \mathbf{a}) \geq s$ for each $\mathbf{a} \in \mathbb{F}_{q}^{m}$, which implies that $P=0$, as desired.

\subsection{Local List-Decoding}

The list-decoding algorithm for multivariate multiplicity codes from the Johnson radius can be used to give a local list-decoding algorithm for multivariate multiplicity codes upto the Johnson radius. Since the definition of local list-decoding is somewhat involved, we refer the reader to the appendix of Kop12 for the algorithm and its analysis.

\section{$5 \quad$ Encoding}

In this section we discuss encoding algorithms.

Since multiplicity codes are $\mathbb{F}_{q}$-linear subspaces of $\Sigma^{\mathbb{F}_{q}^{m}}$, it is natural to choose an encoding map which is $\mathbb{F}_{q}$-linear. One very natural encoding map to consider is the map:

$$
E: \mathbb{F}_{q}^{\left(\begin{array}{c}
d+m \\
m
\end{array}\right)} \rightarrow \mathcal{C}
$$

which treats its input as a vector of coefficients of monomials for a polynomial $P \in \mathbb{F}_{q}[\mathbf{X}]$ of degree at most $d$, and outputs $\operatorname{Enc}_{s, d, m, q}(P)$. It is well known that the task of computing $E$, namely evaluating a given polynomial and all its derivatives of order at most $s$ at all points of $\mathbb{F}_{q}^{m}$ can be performed in near-linear time $O\left(\left(d^{m}+q^{m}\right) \cdot\left(\begin{array}{c}m+s \\ m\end{array}\right) \cdot \log \left(d^{m}+q^{m}\right)\right)$.

However, for the purposes of local decoding, it will be important to choose the encoding map $E: \Sigma_{0}^{k} \rightarrow \mathcal{C} \subseteq$ $\Sigma^{n}$ a bit more carefully. The goal is to have the encoding be systematic; i.e., to have the symbols of the message appear as symbols (or parts of symbols) of its encoding. Once we have such an encoding map, a local correction algorithm immediately gives us a local decoding algorithm.

Such a systematic encoding map can be chosen by giving an interpolating set. Concretely, for a given $s, d, m, q$, we want a set $S \subseteq \mathbb{F}_{q}^{m} \times\{\mathbf{i} \mid \mathrm{wt}(\mathbf{i})<s\}$ such that for every $f: S \rightarrow \mathbb{F}_{q}$, there is exactly one $P\left(X_{1}, \ldots, X_{m}\right) \in \mathbb{F}_{q}\left[X_{1}, \ldots, X_{m}\right]$ of degree at most $d$ such that for each $(\mathbf{a}, \mathbf{i}) \in S, P^{(\mathbf{i})}(\mathbf{a})=f(\mathbf{a}, \mathbf{i})$.

It is easy to see that such sets $S$ exist, and any such set $S$ must have $|S|=\left(\begin{array}{c}d+m \\ m\end{array}\right)$. In order for the so obtained local decoding algorithm to run in sublinear time (assuming the local correction algorithm runs in sublinear time), it will be important that this interpolating set be explicit: given message coordinate $i \in[k]$, we should be able to compute, in time poly $(\log (k))$, the codeword coordinate $j \in[n]$ which contains the $i$ th symbol of the codeword. In Kop12, an explicit such interpolating set was given. 
Theorem 15 ([Kop12]) There exist explicit interpolating sets $S$ as above.

Thus there exist explicit systematic encoding maps for multiplicity codes.

The interpolating sets for multiplicity codes constructed above are in fact simple combinations of interpolating sets for Reed-Muller codes. Furthermore, it is known that there exist interpolating sets for Reed-Muller codes from which polynomial interpolation can be performed in near-linear time. This implies, by inspecting the proof of Theorem 15, that the encoding map described above can be computed in near-linear time.

\section{Discussion}

1. The improved local decoding algorithm given in Section 4.1.2 had two main ideas over the original local decoding algorithm of [KSY11: getting more information from each line, and robustly combining this information across different lines by decoding a multiplicity code.

The first idea is naturally motivated by an incongruity between the three papers: KKSY11] (on local correction of multiplicity codes), GKS13 (on affine invariant codes, local correction of affine invariant codes, and their relationship to bounds on Nikodym sets), and DKSS09] (giving lower bounds on the size of Nikodym sets via the extended method of multiplicities).

A Nikodym set is a set $N \subseteq \mathbb{F}_{q}^{m}$ such that for every $\mathbf{a} \in \mathbb{F}_{q}^{m}$, there is some line $L \subseteq \mathbb{F}_{q}^{m}$ passing through a, such that $L \backslash\{\mathbf{a}\} \subseteq N$ (i.e., the entire line, except possibly a, is contained in $N$ ). In [GKS13] it was noted that lower bounds on Nikodym sets follow from the existence of algebraic error-correcting codes that can be locally corrected at $\mathbf{a} \in \mathbb{F}_{q}^{m}$ by querying a line through $\mathbf{a}$. In [DKSS09], the extended method of multiplicities (which in retrospect can be interpreted in the language of multiplicity codes) was used to show a lower bound of $\left(\frac{q}{2}\right)^{m}$ on the size of Nikodym sets. On the other hand, if one tried to use the original local correction algorithm of [KSY11] for multiplicity codes to prove a lower bound on Nikodym sets (via the GKS13 connection), we would get a significantly weaker bound than the bound of DKSS09]. All this suggests that there should be a better algorithm for local correction of multiplicity codes, and (looking at the details) a way to get more information from each line.

The second idea is motivated by a different incongruity: the local correction algorithm of KSY11] combines information from different lines by decoding a Reed-Muller code, but there seems to be no reason for the $s=1$ case of multiplicity codes (i.e., Reed-Muller codes) to receive preferential treatment amongst all possibilities for $s$. The new algorithm resolves this incongruity, and lets us use fewer lines for the local decoding.

2. The decoding algorithm described in Section 4.1.4 based on $m$ linearly independent lines was motivated by another problem in the combinatorial geometry: the joints problem GK10, KSS10. Alex Vardy and Abdul Basit independently suggested to me that bounds on the joints problem could potentially be improved using the method of multiplicities. The $m$-line decoding algorithm for multiplicity codes can be used to give a multiplicity-enhanced proof of the KSS10] bound on the joints problem; unfortunately this variant does not improve on the bounds.

A brief outline of the argument goes as follows. One first interpolates a low degree polynomial vanishing with multiplicity at least $a$ at the joints of interest (for some large $a$ ). Then one argues that this polynomial vanishes at each line of the collection with multiplicity at least some $c$. Then using Lemma 12, we deduce that this polynomial actually vanishes at each of the joints with multiplicity at least $c^{\prime} \gg a$. This leads to a contradiction, unless the number of joints is large.

3. The Reed-Muller codes over small fields and in many variables have been very influential. The analogous multiplicity code would be based on order $s$ evaluations of polynomials over $\mathbb{F}_{q}$ of total degree at most $d$ and individual degree at most $s q-1$. It would be very interesting to study properties of these codes, and to see if they have any coding/combinatorial applications. 
We note a curious example of how things change when the individual degrees are bounded: a polynomial $P\left(X_{1}, \ldots, X_{m}\right) \in \mathbb{F}_{2}\left[X_{1}, \ldots, X_{m}\right]$ of individual degree at most 1 vanishes at a point $\mathbf{a} \in \mathbb{F}_{2}^{m}$ with multiplicity at least $s$ if and only if $P$ vanishes at all points of $\mathbb{F}_{2}^{n}$ which are at a Hamming distance at most $s-1$ from $\mathbf{a}$.

4. There are various interesting variations/cousins of Reed-Solomon and Reed-Muller codes which are interesting from the coding perspective. These include algebraic-geometric codes, $\mathrm{BCH}$ codes, projective Reed-Muller codes, Grassman codes, etc. It would be interesting to investigate multiplicity-based generalizations of these codes. For algebraic-geometric codes, some such investigations were made (in the 1-dimensional case, analogous to Reed-Solomon codes) by Rosenbloom-Tsfasman [RT97, Xing [Xin03] and Nielsen [Nie01].

\section{Open Questions}

We conclude with a list of some interesting open questions. Some of these questions are open even in the classical $s=1$ case (Reed-Solomon codes and Reed-Muller codes).

1. What is the list-decoding radius for univariate multiplicity codes? In other words, what is the largest fraction $\eta$ of errors from which univariate multiplicity codes of distance $\delta$ and block-length $n$ can be list-decoded with poly $(n)$ list-size? For general univariate multiplicity codes, this is only known to be true for $\eta \leq 1-\sqrt{1-\delta}$, while for univariate multiplicity codes over prime fields with $s$ sufficiently large, it is known for every $\eta<\delta$.

This question is even open for Reed-Solomon codes.

Here are some related questions. Does the answer depend on the field? Does the answer depend on the set of evaluation points? What happens for multivariate multiplicity codes (again, this is open for multivariate Reed-Muller codes too)?

2. It is an extremely interesting question whether the list-size for list-decoding univariate multiplicity codes over prime fields (as in GW11, Kop12]) needs to be poly $(q)$, or if it can be reduced to a constant independent of $q$.

It is also extremely interesting to know whether the primality of $q$ is essential for the improved listdecoding of multiplicity codes in GW11, Kop12.

3. Can Nielsen's algorithm for list-decoding univariate multiplicity codes upto the Johnson bound be implemented to run in near-linear time. It would also immediately imply a near-linear time global algorithm, and a faster local algorithm, for list-decoding multivariate multiplicity codes upto the Johnson radius. This seems to require some nontrivial adaptation of the ideas of Alekhnovich Ale05, who showed how to list-decode Reed-Solomon codes in near-linear time.

4. Are multiplicity codes locally testable?

5. Are there any applications of multiplicity codes to computational complexity theory? Reed-Solomon codes and Reed-Muller codes have found many celebrated applications, and it would be interesting to see if multiplicity codes can improve on any of them.

6. Are there any practical applications of multiplicity codes? By combining high rate with sublineartime decoding, and also supporting various other efficent operations, multiplicity codes seem to be theoretically practical. Perhaps they really are practical? 


\section{Acknowledgements}

Thanks to Alexander Barg and Oleg Musin for organizing an excellent workshop to celebrate Ilya Dumer's birthday, and for encouraging me to write this article. Many many thanks to Ilya Dumer for being a wonderful friend and mentor for many years, and for getting me hooked on coding theory in the first place.

\section{References}

[Ale05] Michael Alekhnovich. Linear diophantine equations over polynomials and soft decoding of reedsolomon codes. IEEE Transactions on Information Theory, 51(7):2257-2265, 2005.

[BI01] Amos Beimel and Yuval Ishai. Information-theoretic private information retrieval: A unified construction. In ICALP, pages 912-926, 2001.

[Chi76] Francis Y. L. Chin. A generalized asymptotic upper bound on fast polynomial evaluation and interpolation. SICOMP: SIAM Journal on Computing, 5, 1976.

[DKSS09] Zeev Dvir, Swastik Kopparty, Shubhangi Saraf, and Madhu Sudan. Extensions to the method of multiplicities, with applications to Kakeya sets and mergers. In 50th IEEE Symposium on Foundations of Computer Science (FOCS), pages 181-190, 2009.

[GK10] Larry Guth and Nets Hawk Katz. Algebraic methods in discrete analogs of the kakeya problem. Advances in Mathematics, 225(5):2828-2839, 2010.

[GKS13] Alan Guo, Swastik Kopparty, and Madhu Sudan. New affine-invariant codes from lifting. In ITCS, pages 529-540, 2013.

[GR08] Venkatesan Guruswami and Atri Rudra. Explicit codes achieving list decoding capacity: Errorcorrection with optimal redundancy. IEEE Transactions on Information Theory, 54(1):135-150, 2008.

[Guo13] Alan Guo. High rate locally correctable codes via lifting. Electronic Colloquium on Computational Complexity (ECCC), 20:53, 2013.

[GW11] Venkatesan Guruswami and Carol Wang. Optimal rate list decoding via derivative codes. In APPROX-RANDOM, pages 593-604, 2011.

[HKT08] J. W. P. Hirschfeld, G. Korchmaros, and F. Torres. Algebraic Curves over a Finite Field (Princeton Series in Applied Mathematics). Princeton University Press, 2008.

[HOW13] Brett Hemenway, Rafail Ostrovsky, and Mary Wootters. Local correctability of expander codes. In $\operatorname{ICALP}(1)$, pages 540-551, 2013.

[Kop12] Swastik Kopparty. List-decoding multiplicity codes. In Electronic Colloquium on Computational Complexity (ECCC), TR12-044, 2012.

[KSS10] Haim Kaplan, Micha Sharir, and Eugenii Shustin. On lines and joints. Discrete \& Computational Geometry, 44(4):838-843, 2010.

[KSY11] Swastik Kopparty, Shubhangi Saraf, and Sergey Yekhanin. High-rate codes with sublinear-time decoding. In STOC, pages 167-176, 2011.

[Nie01] Rasmus R. Nielsen. List decoding of linear block codes. PhD thesis, Technical University of Denmark, 2001. 
[PV05] Farzad Parvaresh and Alexander Vardy. Correcting errors beyond the Guruswami-Sudan radius in polynomial time. In 46th IEEE Symposium on Foundations of Computer Science (FOCS), pages 285-294, 2005.

[PW04] Ruud Pellikaan and Xin-Wen Wu. List decoding of q-ary Reed-Muller codes. IEEE Transactions on Information Theory, 50(4):679-682, 2004.

[RT97] M Yu Rosenbloom and Michael Anatol'evich Tsfasman. Codes for the m-metric. Problemy Peredachi Informatsii, 33(1):55-63, 1997.

[Str81] Volker Strassen. The computational complexity of continued fractions. In Proceedings of the fourth ACM symposium on Symbolic and algebraic computation, pages 51-67. ACM, 1981.

[Sud01] Madhu Sudan. Notes on rational function interpolation. Lecture notes for Algorithmic Introduction to Coding Theory, 2001.

[Xin03] Chaoping Xing. Nonlinear codes from algebraic curves improving the Tsfasman-Vladut-Zink bound. IEEE Transactions on Information Theory, 49(7):1653-1657, 2003. 